\title{
Busy Period and Busy Cycle Distributions and Parameters for a Particular M/G/œ Queue System
}

\author{
Manuel Alberto M. Ferreira ", Marina Andrade
}

Department of Quantitative Methods, University Institute of Lisbon, Lisbon, 1649-026, Portugal

\begin{abstract}
Solving a Riccati equation a collection of service times distributions, very general, is determined. For it the $M / G / \infty$ queue busy period and busy cycle probabilistic study, very comfortable, is performed. In addition the properties of that distributions collection are deduced and also presented.
\end{abstract}

Keywords Riccati Equation, Probability Distributions, $M / G / \infty$, Busy Period, Busy Cycle

\section{Introduction}

In the $M / G / \infty$ queue system the costumers arrive according to a Poisson process at rate $\lambda$. They receive a service which length is a positive random variable with distribution function $\mathrm{G}($.) and mean $\alpha$. There are always available servers. The traffic intensity is $\rho=\lambda \alpha$.

At the operation of a $M / G / \infty$ queue system, such as in any other queue system, there is a sequence of idle periods and busy periods. An idle period followed by a busy period is called a busy cycle.

When applying this queue system to real problems, the busy period and the busy cycle length probabilistic study is very important. Along this work it is shown that the solution of the problem may be obtained solving a Riccati equation. The solution is a collection of service distributions for which both the busy period and the busy cycle have lengths with quite simple distributions, generally related with exponential distributions and the degenerated at the origin one.

Some results for that collection of service distributions are also presented.

\section{A Riccati Equation Important in the $M / G / \infty$ Occupation Study}

Defining $\beta(t)=(1-p) h(t)-\lambda p-\lambda(1-p) G(t), 0 \leq p<1 \quad$, where $h(t)=\frac{g(t)}{1-G(t)}$ with $g(t)=G^{\prime}(t)$ it is obtained

$$
\begin{aligned}
& \frac{d G(t)}{d t}=-\lambda G^{2}(t)-\left(\frac{\lambda p+\beta(t)}{1-p}-\lambda\right) G(t) \\
& +\frac{\lambda p+\beta(t)}{1-p}, \quad 0 \leq p<1
\end{aligned}
$$

* Corresponding author:

manuel.ferreira@iscte.pt (Manuel Alberto M. Ferreira)

Published online at http://journal.sapub.org/ajms

Copyright (C) 2012 Scientific \& Academic Publishing. All Rights Reserved a Riccati equation which solution is

$$
\begin{gathered}
G(t)=1-\frac{1}{\lambda} \frac{\left(1-e^{-\rho}\right) e^{-\lambda t-\int_{0}^{t} \frac{\lambda p+\beta(u)}{1-p} d u}}{\int_{0}^{\infty} e^{-\lambda w-\int_{0}^{w} \frac{\lambda p+\beta(u)}{1-p} d u} d w-}, \\
t \geq 0, \quad-\lambda \leq \frac{\int_{0}^{t} \frac{\lambda p+\beta(u)}{1-p} d u}{t} \leq \frac{\lambda}{e^{\rho}-1}, 0 \leq p<1
\end{gathered}
$$

see [1]. Note that, for $t \geq 0, G(t)=1$ is a solution.

\section{Busy Period and Busy Cycle Study}

After (2) and considering

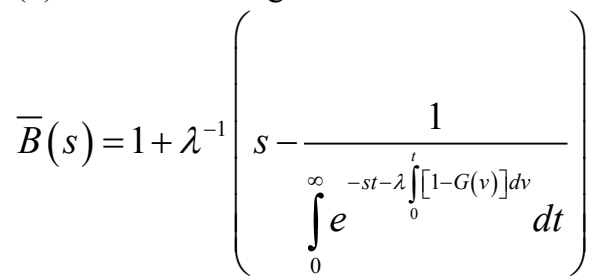

that is the $M / G / \infty$ system busy period Laplace transform, see [2], it is obtained

$$
\begin{gathered}
\bar{B}(s)=\frac{1-(s+\lambda)(1-G(0)) L\left[e^{-\lambda t-\int_{0}^{t} \frac{\lambda p+\beta(u)}{1-p} d u}\right]}{1-\lambda(1-G(0)) L\left[e^{-\lambda t-\int_{0}^{t} \frac{\lambda p+\beta(u)}{1-p} d u}\right]}, \\
-\lambda \leq \frac{\int_{0}^{t} \frac{\lambda p+\beta(u)}{1-p} d u}{t} \leq \frac{\lambda}{e^{\rho}-1}, \quad 0 \leq p<1
\end{gathered}
$$




$$
G(0)=\frac{\lambda \int_{0}^{\infty} e^{-\lambda w-\int_{0}^{w} \frac{\lambda p+\beta(u)}{1-p} d u} d w+e^{-\rho}-1}{\lambda \int_{0}^{\infty} e^{-\lambda w-\int_{0}^{w} \frac{\lambda p+\beta(u)}{1-p} d u} d w} .
$$

After expression (4) it is possible to compute $\frac{1}{s} \bar{B}(s)$ which inversion gives

$$
\begin{aligned}
& B(t)=\left(1-(1-G(0))\left(\begin{array}{l}
e^{-\lambda t-\int_{0}^{t} \frac{\lambda p+\beta(u)}{1-p} d u} \\
\left.+\lambda \int_{0}^{t} e^{-\lambda w-\int_{0}^{w} \frac{\lambda p+\beta(u)}{1-p} d u} d w\right)
\end{array}\right) *\right. \\
& \sum_{n=0}^{\infty} \lambda^{n}(1-G(0))^{n}\left(e^{-\lambda t-\int_{0}^{t} \frac{\lambda p+\beta(u)}{1-p} d u}\right)^{*}, \\
& -\lambda \leq \frac{\int_{0}^{t} \frac{\lambda p+\beta(u)}{1-p} d u}{t} \leq \frac{\lambda}{e^{\rho}-1}, 0 \leq p<1
\end{aligned}
$$

for the $M / G / \infty$ system busy period length distribution function, being $*$ the convolution operator.

If $\beta(t)=\beta$ (constant), (2) becomes, see[3],

$$
\begin{gathered}
G(t)=1-\frac{\left(1-e^{-\rho}\right)\left(\lambda+\frac{\lambda p+\beta}{1-p}\right)}{\lambda e^{-\rho}\left(e^{\left(\lambda+\frac{\lambda p+\beta}{1-p}\right) t}-1\right)+\lambda}, \\
t \geq 0,-\lambda \leq \frac{\lambda p+\beta}{1-p} \leq \frac{\lambda}{e^{\rho}-1}, 0 \leq p<1
\end{gathered}
$$

and (5)

$$
\begin{aligned}
& B^{\beta}(t)=1-\frac{\lambda+\frac{\lambda p+\beta}{1-p}}{\lambda}\left(1-e^{-\rho}\right) e^{-e^{-\rho}\left(\lambda+\frac{\lambda p+\beta}{1-p}\right) t} \\
& t \geq 0,-\lambda \leq \frac{\lambda p+\beta}{1-p} \leq \frac{\lambda}{e^{\rho}-1}, 0 \leq p<1
\end{aligned}
$$

So, if the service distribution is given by (6), the busy period distribution function is the one of the mixture of a degenerated distribution at the origin with an exponential.

For $\beta=-\lambda, 0 \leq p<1$,

$$
G(t)=1, t \geq 0 \text { and } B^{\beta}(t)=1, t \geq 0
$$

that is if the service distribution is degenerated at the origin with probability 1 it happens the same with the busy period distribution.

Finally note that for $\beta=\lambda \frac{1-p e^{\rho}}{e^{\rho}-1}, 0 \leq p<1$

$$
B^{\beta}(t)=1-e^{-\frac{\lambda}{e^{\beta}-1} t}, t \geq 0
$$

(purely exponential). And $B(t)$ given by (5) satisfies

$$
B(t) \geq 1-e^{-\frac{\lambda}{e^{\rho}-1} t}, t \geq 0 .
$$

Calling $\bar{Z}(s)$ the busy cycle length Laplace transform, and noting that the $M / G / \infty$ queue idle period is exponentially distributed with parameter $\lambda$ - as it happens for any queue system with Poisson arrivals at rate $\lambda$ - and that the idle period and the busy period are independent for the $M / G / \infty$ queue, see[4], it is obtained

$$
\bar{Z}(s)=\frac{\lambda}{\lambda+s} \bar{B}(s)
$$

After (4) and (8) it is possible to compute $(1 / s) \bar{Z}(s)$ which inversion gives

$$
\begin{aligned}
& Z(t)=\left(\lambda e^{-\lambda t}\right) *\left(\begin{array}{l}
1-(1-G(0)) \\
\left(e^{-\lambda t-\int_{0}^{t} \frac{\lambda p+\beta}{1-p} d u}+\lambda \int_{0}^{t} e^{-\lambda w-\int_{0}^{w} \frac{\lambda p+\beta}{1-p} d u} d w\right)
\end{array}\right) * \\
& * \sum_{n=0}^{\infty} \lambda^{n}(1-G(0))^{n}\left(e^{-\lambda t-\int_{0}^{1} \frac{\lambda p+\beta}{1-p} d u}\right)^{* n}, \\
& -\lambda \leq \frac{\int_{0}^{t} \frac{\lambda p+\beta}{1-p} d u}{t} \leq \frac{\lambda}{e^{\rho}-1}, 0 \leq p<1
\end{aligned}
$$

for the busy cycle distribution function.

If $\beta(t)=\beta$ (constant) (9) becomes

$$
\begin{aligned}
& Z^{\beta}(t)=1-\frac{\left(1-e^{-\rho}\right)\left(\lambda+\frac{\lambda p+\beta}{1-p}\right)}{\lambda-e^{-\rho}\left(\lambda+\frac{\lambda p+\beta}{1-p}\right)} e^{-e^{-\rho}\left(\lambda+\frac{\lambda p+\beta}{1-p}\right) t}+ \\
& \frac{\frac{\lambda p+\beta}{1-p}}{\lambda-e^{-\rho}\left(\lambda+\frac{\lambda p+\beta}{1-p}\right)} e^{-\lambda t}, \\
& t \geq 0, \quad-\lambda \leq \frac{\lambda p+\beta}{1-p} \leq \frac{\lambda}{e^{\rho}-1}, \quad 0 \leq p<1
\end{aligned}
$$

So, if the service time distribution function is given by (6) the busy cycle distribution function is the mixture of two exponential distributions.

Note that, for $\beta=\lambda \frac{1-p e^{\rho}}{e^{\rho}-1}, 0 \leq p<1$

$$
Z^{\beta}(t)=1-\frac{\left(e^{\rho}-1\right) e^{-\frac{\lambda}{e^{\rho}-1} t}-e^{-\lambda t}}{e^{\rho}-2}, t \geq 0 .
$$

And $Z(t)$, given by (9), satisfies

$$
Z(t) \geq 1-\frac{\left(e^{\rho}-1\right) e^{-\frac{\lambda}{e^{\rho}-1} t}-e^{-\lambda t}}{e^{\rho}-2}, t \geq 0
$$

Note that (11) is coherent even for $\rho=\log 2$ since 


$$
\begin{aligned}
& \lim _{\rho \rightarrow \log 2}\left(1-\frac{\left(e^{\rho}-1\right) e^{-\frac{\lambda}{e^{\rho}-1} t}-e^{-\lambda t}}{e^{\rho}-2}\right)= \\
& \lim _{\rho \rightarrow \log 2} \frac{e^{\rho}-2-\left(e^{\rho}-1\right) e^{-\frac{\lambda}{e^{\rho}-1}}-e^{-\lambda t}}{e^{\rho}-2}=1-(1+\lambda t) e^{-\lambda t} .
\end{aligned}
$$

For $\beta=-\lambda, 0 \leq p<1$

$$
Z^{\beta}(t)=1-e^{-\lambda t}, t \geq 0 .
$$

Finally, also for $Z(t)$ given by (9),

$$
Z(t) \leq 1-e^{-\lambda t}, \quad t \geq 0
$$

\section{Busy Period and Busy Cycle Parameters}

As for the busy period moments, calling $B$ the busy period random variable, note that (3) is equivalent to, see [5],

being

$$
(\bar{B}(s)-1)(C(s)-1)=\lambda^{-1} s C(s) \text {, }
$$

$$
C(s)=\int_{0}^{\infty} e^{-s t-\lambda \int_{0}^{t}[1-G(v)] d v} \lambda(1-G(t)) d t .
$$

Differentiating $n$ times, using Leibnitz's formula and making $s=0$, results

$$
\begin{aligned}
& E\left[B^{n}\right]=(1)^{n+1}\left\{\frac{e^{\rho}}{\lambda} n C^{(n-1)}(0)-e^{\rho} \sum_{\rho=1}^{n-1}(-1)^{n-p}\left(\begin{array}{l}
n \\
p
\end{array}\right) \cdot E\left[B^{n-p}\right] C^{(p)}(0)\right\}, \\
& n=1,2, \ldots
\end{aligned}
$$

where

$$
\begin{aligned}
& C^{(n)}(0)=\int_{0}^{\infty}(-t)^{n} e^{-\lambda \int_{0}^{t}[1-G(v)] d v} \lambda(1-G(t)) d t, \\
& n=0,1,2, .
\end{aligned}
$$

The expression (13) gives a recursive method to compute $E\left[B^{n}\right], n=1,2, \ldots$ as a function of $C^{(n)}(0), n=0,1,2, \ldots$

So it is possible to write exact expressions for the moments centred at the origin.

Making $n=1$ it is obtained

$$
E[B]=\frac{e^{\rho}-1}{\lambda}
$$

as it is well known, independently of the service time distribution.

For the busy cycle moments, if $\mathrm{Z}$ is the busy cycle random variable,

$$
E\left[Z^{n}\right]=\sum_{p=0}^{n}\left(\begin{array}{l}
n \\
p
\end{array}\right) E\left[B^{p}\right] \cdot \frac{(n-p) !}{\lambda^{n-p}}
$$

The $M|G| \infty$ queue busy period "peak" is the Laplace transform value at $1 / \alpha$, see[6]. It is a parameter that characterizes the busy period distribution and contains information about all its moments.

For the collection of service distributions (2) the "peak", called $p i$, is

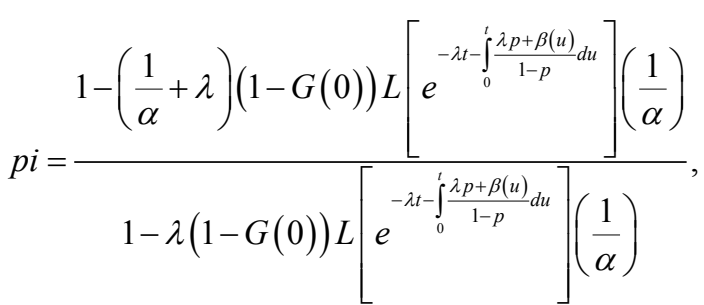

$$
-\lambda \leq \frac{\int_{0}^{t} \frac{\lambda p+\beta(u)}{1-p} d u}{t} \leq \frac{\lambda}{e^{\rho}-1}, \quad 0 \leq p<1
$$

For $\beta(t)=\beta$ (constant), see[7],

$$
\begin{aligned}
& p i=\frac{e^{-\rho}(\lambda+\beta)(\rho+1)-\lambda p-\beta}{\lambda\left(e^{-\rho}(\rho+\alpha \beta)+1-p\right)}, \\
& -\lambda \leq \beta \leq \frac{\lambda\left(1-p e^{\rho}\right)}{e^{\rho}-1}, 0 \leq p<1
\end{aligned}
$$

In[6] it is also introduced another measure: the "modified peak" got after the "peak" taking out the terms that are permanent for the busy period in different service distributions and putting over the common part. Call it $q i$. It is easy to verify that $q i=p i \frac{\rho}{e^{\rho}-\rho-1}+1$ and, so, for the distributions given by collection (2) with $\beta(t)=\beta$ (constant), so collection (6), see again[7],

$$
\begin{aligned}
& q i=\frac{e^{-\rho}(\lambda+\beta)(\rho+1)-\lambda p+\beta}{\lambda\left(e^{-\rho}(\rho+\alpha \beta)+1-p\right)} \frac{\rho}{e^{\rho}-\rho-1}+1, \\
& -\lambda \leq \beta \leq \frac{\lambda\left(1-p e^{\rho}\right)}{e^{\rho}-1}, 0 \leq p<1
\end{aligned}
$$

For the busy cycle of the $M|G| \infty$ queue the "peak", see[8], called now $p i^{\prime}$, for the service distributions given by the collection (2) is

$$
p i^{\prime}=\frac{\rho}{\rho+1} \frac{1-\left(\frac{1}{\alpha}+\lambda\right)(1-G(0)) L\left[e^{-\lambda t-\int_{0}^{t} \frac{\lambda p+\beta(u)}{1-p} d u}\right]\left(\frac{1}{\alpha}\right)}{1-\lambda(1-G(0)) L\left[e^{-\lambda t-\int_{0}^{t} \frac{\lambda p+\beta(u)}{1-p} d u}\right]\left(\frac{1}{\alpha}\right)},
$$

$-\lambda \leq \frac{\int_{0}^{t} \frac{\lambda p+\beta(u)}{1-p} d u}{t} \leq \frac{\lambda}{e^{\rho}-1}, \quad 0 \leq p<1$

With $\beta(t)=\beta$ (constant), see[7],

$$
\begin{aligned}
& p i^{\prime}=\alpha \frac{e^{-\rho}(\lambda+\beta)(\rho+1)-\lambda p-\beta}{(\rho+1)\left(e^{-\rho}(\rho+\alpha \beta)+1-p\right)}, \\
& -\lambda \leq \beta \leq \frac{\lambda\left(1-p e^{\rho}\right)}{e^{\rho}-1}, 0 \leq p<1
\end{aligned}
$$

And the "modified peak", called now $q i^{\prime}$, given by $p i^{\prime} \frac{\rho}{e^{\rho}-\rho}+1$, for the service distributions given by the collection (6) is 


$$
\begin{aligned}
& q i^{\prime}=\alpha \frac{e^{-\rho}(\lambda+\beta)(\rho+1)-\lambda p-\beta}{(\rho+1)\left(e^{-\rho}(\rho+\alpha \beta)+1-p\right)} \frac{\rho}{e^{\rho}-\rho}+1, \\
& -\lambda \leq \beta \leq \frac{\lambda\left(1-p e^{\rho}\right)}{e^{\rho}-1}, 0 \leq p<1
\end{aligned}
$$

The busy cycle renewal function value of the $M|G| \propto$ queue, at the instant $t$, gives the mean number of busy periods that begin in $[0, t]$, see [9]. Calling it $R(t)$ :

$$
R(t)=e^{-\lambda \int_{0}^{t}[1-G(v)] d v}+\lambda \int_{0}^{t} e^{-\lambda \int_{0}^{u}[1-G(v)] d v} d u
$$

If the service time is a random variable with distribution function given by a member of the collection (6)

$$
\begin{aligned}
& R(t)=e^{-\rho}(1+\lambda t)+\left(1-e^{-\rho}\right) \frac{\lambda p+\beta}{\lambda+\beta} e^{-\left(\lambda+\frac{\lambda p+\beta}{1-p}\right) t}+ \\
& \left(1-e^{-\rho}\right) \frac{\lambda p+\beta}{\lambda+\beta},-\lambda \leq \beta \leq \frac{\lambda\left(1-p e^{\rho}\right)}{e^{\rho}-1}, 0 \leq p<1
\end{aligned}
$$

\section{Collection (2) Distributions Moments Computation}

If, in (2), $G_{i}(t)$ is the solution associated to $\rho_{i}$, $i=1,2,3,4$ it is easy to see that

$$
\frac{G_{4}(t)-G_{2}(t)}{G_{4}(t)-G_{1}(t)} \cdot \frac{G_{3}(t)-G_{1}(t)}{G_{3}(t)-G_{2}(t)}=\frac{e^{-\rho_{4}}-e^{-\rho_{2}}}{e^{-\rho_{4}}-e^{-\rho_{1}}} \cdot \frac{e^{-\rho_{3}}-e^{-\rho_{1}}}{e^{-\rho_{3}}-e^{-\rho_{2}}}
$$

as it had to happen since the differential equation considered is a Riccati one.

And computing,

$$
\begin{aligned}
& \int_{0}^{\infty}[1-G(t)] d t= \\
& \int_{0}^{\infty} \frac{1}{\lambda} \frac{\left(1-e^{-\rho}\right) e^{-\lambda t-\int_{0}^{t} \frac{\lambda p+\beta(u)}{1-p} d u}}{\int_{0}^{\infty} e^{-\lambda w-\int_{0}^{w} \frac{\lambda p+\beta(u)}{1-p} d u} d w-\left(1-e^{-\rho}\right) \int_{0}^{t} e^{-\lambda w-\int_{0}^{w} \frac{\lambda p+\beta(u)}{1-p} d u} d w} d t=\alpha
\end{aligned}
$$

in accordance to the fact that (2) is a collection of positive random variables distribution functions.

The density associated to $G(t)$ given by (2) is

$$
\begin{aligned}
& g(t)=-\frac{1}{\lambda}\left[\frac{A^{\prime \prime}(t)}{B-A(t)}+\frac{\left[A^{t}(t)\right]^{2}}{[B-A(t)]^{2}}\right], \\
& t \geq 0, \quad-\lambda \leq \frac{\int_{0}^{t} \frac{\lambda p+\beta(u)}{1-p} d u}{t} \leq \frac{\lambda}{e^{\rho}-1}, 0 \leq p<1
\end{aligned}
$$

where

$$
A(t)=\left(1-e^{-\rho}\right) \int_{0}^{t} e^{-\lambda w-\int_{0}^{w} \frac{\lambda p+\beta(u)}{1-p} d u} d w
$$

and

$$
B=\int_{0}^{\infty} e^{-\lambda w-\int_{0}^{w} \frac{\lambda_{p}+\beta(u)}{1-p} d u} d w .
$$

If $\beta(t)=\beta$ (constant), see[7],

$$
\begin{aligned}
& g(t)=\frac{\left(1-e^{-\rho}\right) e^{-\rho}\left(\lambda+\frac{\lambda p+\beta}{1-p}\right)^{2} e^{-\left(\lambda+\frac{\lambda p+\beta}{1-p}\right) t}}{\lambda\left[e^{-\rho}+\left(1-e^{-\rho}\right) e^{-\left(\lambda+\frac{\lambda p+\beta}{1-p}\right) t}\right]^{2}}, \\
& t>0,-\lambda \leq \beta \leq \frac{\lambda\left(1-p e^{-\rho}\right)}{e^{\rho}-1}, 0 \leq p<1
\end{aligned}
$$

So,

$$
\begin{aligned}
& \int_{0}^{\infty} t^{n} g(t) d t=\frac{\left(1-e^{-\rho}\right) e^{-\rho}\left(\lambda+\frac{\lambda p+\beta}{1-p}\right)^{2}}{\lambda} \times \\
& \int_{0}^{\infty} t^{n} \frac{e^{-\left(\lambda+\frac{\lambda p+\beta}{1-p}\right) t}}{\left[e^{-\rho}+\left(1-e^{-\rho}\right) e^{-\left(\lambda+\frac{\lambda p+\beta}{1-p}\right) t}\right]^{2}} d t
\end{aligned}
$$

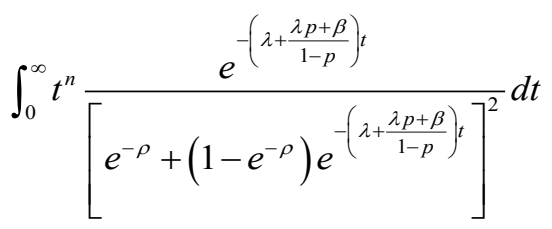

But,

$$
\begin{aligned}
& \geq \int_{0}^{\infty} t^{n} e^{-\left(\lambda+\frac{\lambda p+\beta}{1-p}\right) t} d t= \\
& \frac{1}{\lambda+\frac{\lambda p+\beta}{1-p}} \frac{n !}{\left(\lambda+\frac{\lambda p+\beta}{1-p}\right)^{n}}, \beta \neq-\lambda .
\end{aligned}
$$

And,

$$
\begin{aligned}
& \int_{0}^{\infty} t^{n} \frac{e^{-\left(\lambda+\frac{\lambda p+\beta}{1-p}\right) t}}{\left[e^{-\rho}+\left(1-e^{-\rho}\right) e^{-\left(\lambda+\frac{\lambda p+\beta}{1-p}\right) t}\right]^{2}} d t \leq \\
& e^{2 \rho} \int_{0}^{\infty} t^{n} e^{-\left(\lambda+\frac{\lambda p+\beta}{1-p}\right) t} d t= \\
& \frac{e^{2 \rho}}{\lambda+\frac{\lambda p+\beta}{1-p}} \frac{n !}{\left(\lambda+\frac{\lambda p+\beta}{1-p}\right)^{n}}, \beta \neq-\lambda .
\end{aligned}
$$

So, calling $\mathrm{T}$ the random variable corresponding to $G(t)$ :

$$
\begin{aligned}
& \frac{\left(1-e^{-\rho}\right) e^{-\rho}}{\lambda} \frac{n !}{\left(\lambda+\frac{\lambda p+\beta}{1-p}\right)^{n-1}} \leq \mathrm{E}\left[\mathrm{T}^{n}\right] \\
& \leq \frac{e^{\rho}-1}{\lambda} \frac{n !}{\left(\lambda+\frac{\lambda p+\beta}{1-p}\right)^{n-1}}, \\
& -\lambda<\beta \leq \frac{\lambda\left(1-p e^{-\rho}\right)}{e^{\rho}-1}, 0 \leq p<1, n=1,2, .
\end{aligned}
$$




\section{Notes:}

The expression (29), giving bounds for $\mathrm{E}\left[\mathrm{T}^{n}\right]$, guarantees its existence,

For $n=1$ the expression (29) is useless because $\mathrm{E}[\mathrm{T}]=\alpha$. Note, curiously, that the upper bound is $\frac{e^{\rho}-1}{\lambda}$, the $M / G / \infty$ system busy period mean value,

For $n=2$, subtracting to both bounds, $\alpha^{2}$ from expression (29) result bounds for $\operatorname{VAR}[\mathrm{T}]$,

For $\beta=-\lambda, \mathrm{E}\left[\mathrm{T}^{n}\right]=0, n=1,2, \ldots$, evidently.

See, however, that (6) can be put like:

$$
\begin{aligned}
& G(t)=\frac{1+\frac{\frac{\lambda p+\beta}{1-p}}{\lambda}\left(1-e^{\rho}\right) e^{-\left(\lambda+\frac{\lambda p+\beta}{1-p}\right) t}}{1-\left(1-e^{\rho}\right) e^{-\left(\lambda+\frac{\lambda p+\beta}{1-p}\right) t}}, \\
& t \geq 0,-\lambda \leq \beta \leq \frac{\lambda\left(1-p e^{\rho}\right)}{e^{\rho}-1}, 0 \leq p<1
\end{aligned}
$$

And, for $\rho<\log 2$,

$$
\begin{aligned}
& G(t)=\left(1+\frac{\frac{\lambda p+\beta}{1-p}}{\lambda}\left(1-e^{\rho}\right) e^{-\left(\lambda+\frac{\lambda p+\beta}{1-p}\right) t}\right) . \\
& \sum_{k=0}^{\infty}\left(1-e^{\rho}\right)^{k} e^{-k\left(\lambda+\frac{\lambda p+\beta}{1-p}\right) t}, \\
& t \geq 0,-\lambda \leq \beta \leq \frac{\lambda\left(1-p e^{\rho}\right)}{e^{\rho}-1}, 0 \leq p<1
\end{aligned}
$$

After (31) the T Laplace Transform for $\rho<\log 2$, can be easily derived. And, so,

- For $\rho<\log 2$

$$
\begin{aligned}
& \mathrm{E}\left[\mathrm{T}^{n}\right]=-\left(1+\frac{\frac{\lambda p+\beta}{1-p}}{\lambda}\right) n ! \sum_{k=1}^{\infty} \frac{\left(1-e^{\rho}\right)^{k}}{k\left(\lambda+\frac{\lambda p+\beta}{1-p}\right)^{n}}, \\
& -\lambda<\beta \leq \frac{\lambda\left(1-p e^{\rho}\right)}{e^{\rho}-1}, 0 \leq p<1, n=1,2, \ldots
\end{aligned}
$$

Notes:

$$
\begin{aligned}
& \mathrm{E}[\mathrm{T}]=-\left(1+\frac{\frac{\lambda p+\beta}{1-p}}{\lambda}\right)_{k=1}^{\infty} \frac{\left(1-e^{\rho}\right)^{k}}{k\left(\lambda+\frac{\lambda p+\beta}{1-p}\right)}= \\
& \frac{1}{\lambda} \sum_{k=1}^{\infty}(-1)^{k+1} \frac{\left(1-e^{\rho}\right)}{k}=\frac{1}{\lambda} \log e^{\rho}=\frac{\rho}{\lambda}=\alpha
\end{aligned}
$$

For $n \geq 2$ only a finite number of parcels in the infinite sum must be taken. Calling $M$ this number, to get an error lesser than $\varepsilon$ it is necessary to have simultaneously

$$
\mathrm{M}>\frac{1}{\lambda+\frac{\lambda p+\beta}{1-p}}-1,
$$

$$
\mathrm{M}>\log _{\left(e^{\rho}-1\right)} \frac{\varepsilon e^{\rho} \lambda}{n !\left(\lambda+\frac{\lambda p+\beta}{1-p}\right)}-1 .
$$

So it is evident now that this distributions collection moments computation is a complex task. This was already true for the study of [10] where the results presented are a particular situation of these ones for $p=0$.

The consideration of the approximation

$$
\begin{aligned}
& \mathrm{E}_{m}^{n}=\sum_{k=1}^{\infty}\left(\frac{k}{m}\right)^{n}\left[G\left(\frac{k}{m}\right)-G\left(\frac{k-1}{m}\right)\right], \\
& -\lambda<\beta \leq \frac{\lambda\left(1-p e^{\rho}\right)}{e^{\rho}-1}, 0 \leq p<1, n=1,2, \ldots
\end{aligned}
$$

may be helpful since $\lim _{m \rightarrow \infty} \mathrm{E}_{m}^{n}=\mathrm{E}\left[\mathrm{T}^{n}\right], n=1,2, \ldots, \operatorname{see}[11]$, that allow the numerical computation of the moments.

\section{Conclusions}

The function $\beta(t)$, defined in 1., that leads to the equation (1), the source of the results presented, is induced by the $M / G / \infty$ transient probabilities monotony study, see[1] and[3]. Being well known the close relations existing among the transient behaviour and the busy period distributions, it is not surprising that the service distribution functions, solutions of (1), give rise to so friendly distributions to the $M / G / \infty$ queue busy period. This is a quite unusual situation, that allows simple computations for the probabilities and parameters of the busy period and busy cycle.

This so simple structure lies on the exponential distribution and the deterministic one. In many of the situations seen there are purely deterministic and exponential distributions, mixtures of deterministic and exponential distributions and mixtures of two exponential distributions. The great presence of the exponential distribution in this context is due certainly to the so friendly properties of the Poisson Process and to the structure of the function resulting from the inversion of the Laplace Transform given by (4).

Even the stochastic processes related with this queue become quite simple. They are very close to the Poisson Process and in certain situations are even Poisson Processes.

In conclusion, all of this results in a very simple expression for the inversion of (4), with the service distributions solutions of (1). In consequence, as it was shown in this work, the distribution functions and the parameters for the busy period and, consequently, for the busy cycle result very simple to compute and interpret.

Many of these results are true for other service distribution functions, in the case of insensibility: parameters that depend on the service distribution only through its mean. Some others may result in good approximations.

Note still that when the distribution functions for $B(t)$ and $Z(t)$ are a little more complicated, simple bounds are presented. 
In the field of Queuing Theory this situation seems unique, there having no news of anything similar.

\section{ACKNOWLEDGEMENTS}

This work was financially supported by FCT through the Strategic Project PEst-OE/EGE/UI0315/2011.

\section{REFERENCES}

[1] M. A. M. Ferreira and M. Andrade, 2011, $M / G / \infty$ queue system transient behavior with time origin at an operation beginning instant and occupation, Journal of Mathematics and Technology, 2(1), 54-60

[2] W. Stadje, 1985, The busy period of the queueing $M / G / \infty$, Journal of Applied Probability, 22, 697-704

[3] M. A. M. Ferreira and M. Andrade, 2010, Looking to a $M / G / \infty$ system occupation through a Riccati equation, Journal of Mathematics and Technology, 1(2), 58-62

[4] L. Takács, An Introduction to Queueing Theory, Oxford University Press, 1962

[5] M. A. M. Ferreira, Comportamento transeunte e período de ocupação de sistemas de filas de espera sem espera, $\mathrm{PhD}$ thesis, ISCTE, Lisbon, Portugal, April 1995

[6] M. A. M. Ferreira, 1997, Um parâmetro caracterizador da distribuição do período de ocupação da fila de espera
$M / G / \infty$, Revista Portuguesa de Gestão II, 99-102

[7] M. A. M. Ferreira and M. Andrade, 2009, $M / G / \infty$ queue system parameters for a particular collection of service time distributions, African Journal of Mathematics and Computer Science Research, 2(7), 138-141

[8] M. A. M. Ferreira, 1999, Application of the "modified peak" to characterize the $M / G / \infty$ queue busy cycle length distribution, Statistical Review, 3(3), 47-56

[9] M. A. M. Ferreira, 2004, $M / G / \infty$ queue busy cycle renewal function for some particular service time distributions, Proceedings of Quantitative Methods in Economics (Multiple Criteria Decision Making XII), Virt. Slovakia

[10] M. A. M. Ferreira, 1998, Momentos de Variáveis Aleatórias com Função de Distribuição dadas pela Colecção $G(t)=1-\frac{\left(1-e^{-\rho}\right)(\lambda+\beta)}{\lambda e^{-\rho}\left(e^{(\lambda+\beta)}-1\right)+\lambda}, t \geq 0, \quad-\lambda \leq \beta \leq \frac{\lambda}{e^{\rho}-1}$. Revista Portuguesa de Gestão, II, 67-69

[11] M. A. M. Ferreira, Redes de Filas de Espera, Master Thesis, IST-UTL, Lisbon, Portugal, Feb 1987

[12] M. A. M. Ferreira and M. Andrade, 2011, Fundaments of theory of queues, International Journal of Academic Research, 3(1, Part II), 427-429

[13] M. A. M. Ferreira and M. Andrade, $2010 M / G / \infty$ queue busy period tail, Journal of Mathematics and Technology, 1 (3), 11-16

[14] M. Andrade, 2010, A note on foundations of probability, Journal of Mathematics and Technology, 1 (1), 96-98 